\title{
Effects of Anesthesia Protocol on Perioperative Outcomes and Costs of Lumbar Microdiscectomies
}

\author{
Ebru TARIKCI KILIC ${ }^{1}$, Sait NADERI ${ }^{2}$ \\ ${ }^{1}$ Umraniye Training and Research Hospital, Department of Anesthesiology, Istanbul, Turkey \\ 2Umraniye Training and Research Hospital, Department of Neurosurgery, Istanbul, Turkey \\ Corresponding author: Ebru TARIKCI KILIC ebru.tarkc@yahoo.com
}

\section{ABSTRACT}

AIM: To compare the effects of spinal anesthesia (SA) and general anesthesia (GA) in lumbar microdiscectomy in terms of their costeffectiveness, and perioperative outcomes.

MATERIAL and METHODS: We randomly allocated 100 consecutive patients who were scheduled to undergo elective lumbar microdiscectomy, into either SA or GA groups.

We recorded and evaluated various parameters, including demographic aspects, body mass index (BMI), perioperative hemodynamics, time elapsed from operating room (OR) entry until incision, operative time, time elapsed from application of the surgical dressing to exiting OR, blood loss, post anesthetic care unit (PACU) time, preoperative and postoperative pain scores, postoperative analgesic requirements, first mobilization time, first oral intake, the length of hospital stay, time to return to work, and perioperative anesthetic costs. The patients, anesthesiologists, and neurosurgeons were handed a questionnaire before discharge to determine their satisfaction with the procedure.

RESULTS: Several variables were found to be better in the SA group: the mean arterial pressure and heart rate changes were significantly lower, and the time elapsed from OR entry until incision, operative time, time elapsed from application of the surgical dressing to exiting OR, PACU time, the length of hospital stay, and time to return to work were shorter; furthermore, the postoperative pain scores, the analgesic requirements, the intraoperative blood loss, and the cost of anesthesia were all lower. Moreover the first mobilization and oral intake occurred earlier; and most significantly, the satisfaction of the patients and surgeons was higher in the SA group. Furthermore, we encountered no complications.

CONCLUSION: Based on our results, we conclude that SA is reliable and clinically successful procedure in lumbar microdiscectomy. KEYWORDS: Anesthetic costs, General anesthesia, Lumbar disc surgery, Spinal anesthesia, Time to return to work

\section{INTRODUCTION}

$\mathrm{B}$ oth general anesthesia (GA) and spinal anesthesia (SA) have been used in lumbar disc herniation surgeries, but currently GA is being used almost exclusively. The preference for GA is not based on clinically established superiority, but instead it appears to be based on the surgeons' and/or anesthesiologists' comfort or experience.

A favorable anesthetic technique must offer a rapid onset, rapid reversal, stable hemodynamics, low blood loss, low postoperative pain levels and few episodes of nausea and vomiting. Notably, SA is comparatively advantageous than GA in terms of these properties. The airway and pulmonary complications that occur in prone-positioned patients receiving GA are fewer with SA $(13,14)$. SA enables operation on awake patients to facilitate the positioning of their arms and chest as needed during the operation, avoiding brachial plexus palsy or pressure necrosis of the face, eyes, and chest wall. Additionally, the postoperative pain control and operating conditions are better with SA besides the lower costs $(15,19,22)$. 
In this study, we aimed to perform a comparative analysis between $G A$ and $S A$ in terms of the effects on the perioperative and postoperative clinical parameters; the cost of anesthetic procedure; and the satisfaction levels of patients, anesthesiologists, and surgeons.

\section{MATERIAL and METHODS}

After obtaining approval from our hospital's ethics committee, we conducted a study on 100 patients with physical status I-III per the American Society of Anesthesiologists (ASA) classification who were aged between 18-65 years and were undergoing elective single-level lumbar disc herniation surgery. All the patients signed the written informed consent forms.

The patients were randomly assigned into two groups based on whether their Turkish Republic identification card numbers' first digit number was either even or odd. Patients with odd first digit were placed into the GA group and those with even first digit into the SA group. The observers who obtained the pain scores and other data were blinded to the study protocol. Caregivers (physicians and nurses) were not blinded, but they did not participate in data collection or interpretation (see flow chart) (Figure 1).

Inclusion criteria were, as follows: lack of response to at least 3 months of physiotherapy, and resistance to nonsteroidal antiinflammatory drugs. The primary indication for the inclusion of cases was unilateral, one-level lumbar disc herniation with positive clinical symptomatology and signs and positive radiological signs. Straight leg raise test result was positive in all cases (ranged between 30 and 60 degrees). There was motor power loss in $20 \%$, and sensory dysfunction in $35 \%$ of all cases.

We excluded patients with significant cardiovascular diseases, seizures, coagulopathy, infection at the site of needling, hypovolemia, bleeding abnormalities, history of allergic reactions to any of the study drugs, intracranial hypertension,

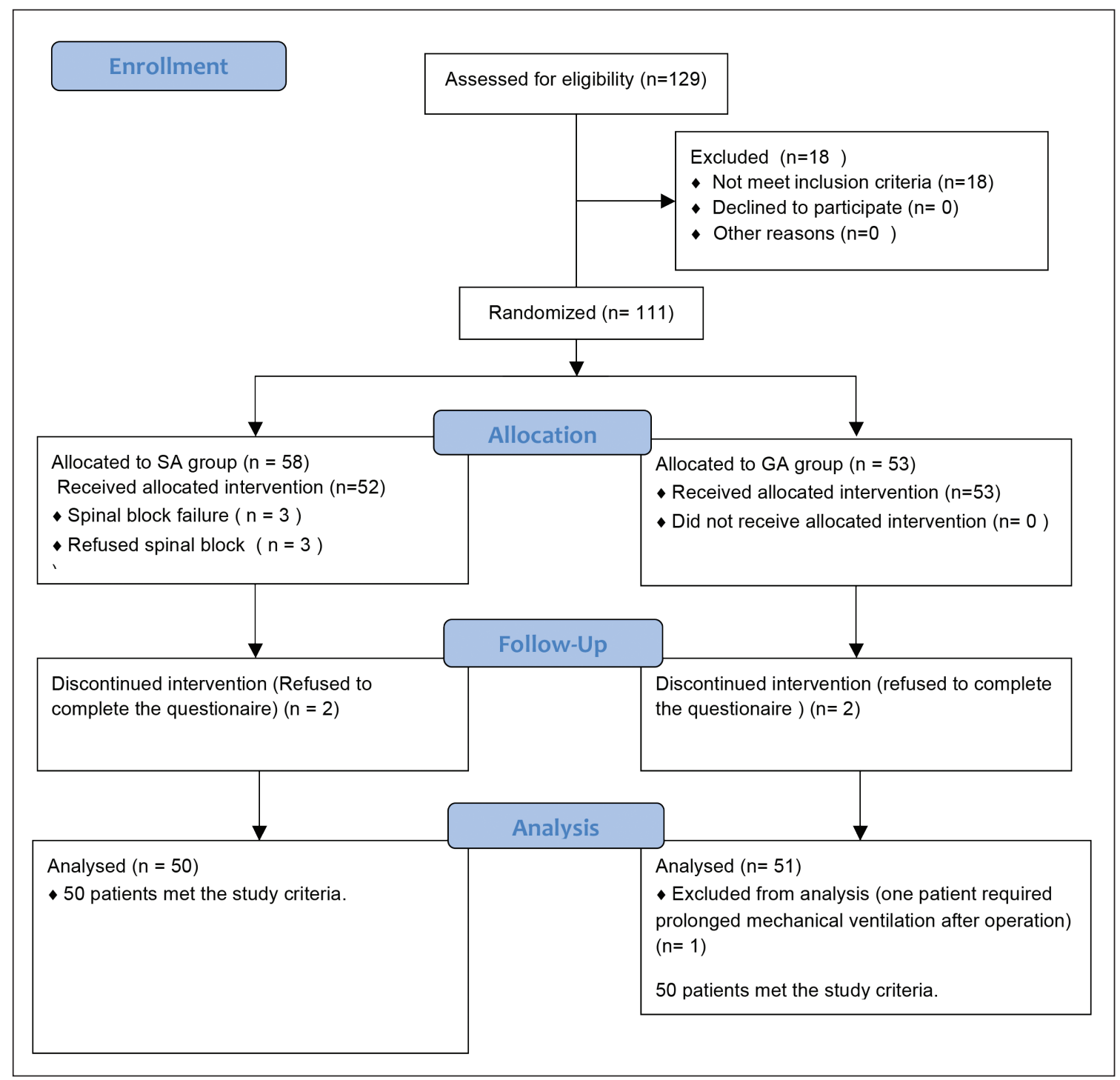

Figure 1: Flow chart of the study protocol. 
and ASA IV-V physical status with life-threatening comorbidities as well as those who refused to undergo SA. The same anesthesia and surgical team performed all the surgical procedures.

\section{Anesthetic Procedure}

For this prospective study, we randomly assigned patients into either the GA or SA group. Upon arrival in the operating room (OR), we introduced an 18-gauge intravenous catheter into the peripheral vein and premedicated each patient with midazolam $0.03-\mathrm{mg} / \mathrm{kg}$ intravenously. The heart rate, peripheral oxygen saturation, and noninvasive blood pressure were routinely monitored every $5 \mathrm{~min}$.

Patients in the SA group received a $10 \mathrm{ml} / \mathrm{kg}$ isotonic solution before the block. SA was administered via a single-injection technique with a 25-gauge spinal needle through a midline approach at the L2-3 or L3-4 interspaces. Following the free flow of cerebrospinal fluid, $3 \mathrm{ml}$ of $0.5 \%$ bupivacaine (Marcaine ${ }^{\circledR}$ Spinal heavy) was injected. As we needed anesthetic effects, both sensory and motor levels were checked until the highest sensory level was reached before the operation. Sensory dermatome level was assessed using the pin prick test every 2 minutes. Motor dermatome level was assessed according to the Bromage scale as follows:

Bromage 0: free movement of legs and feet; 1 : just able to flex knees with free movement of feet; 2 : unable to flex knees but with free movement of feet; 3 : unable to move legs or feet (16).

Once the establishment of the T6-T10 level of SA was confirmed, we moved the patients into the prone position on the operating table. Oxygen was administered through a nasal cannula at a rate of 1-2 L/min. We used additional midazolam, as needed, to achieve sedation. In cases of bradycardia and hypotension, atropine $0.5 \mathrm{mg}$ and ephedrine $5 \mathrm{mg}$ were administered.

On the other hand, the patients undergoing GA were administered propofol $2 \mathrm{mg} / \mathrm{kg}$ and fentanyl $1 \mu \mathrm{g} / \mathrm{kg}$ intravenously for anesthesia induction. Endotracheal intubation was facilitated with intravenous rocuronium bromide $0.5-\mathrm{mg} / \mathrm{kg}$, and anesthesia was maintained with $2 \%$ sevoflurane in $50 \%: 50 \%$ air/oxygen. Following intubation, we placed the patients in the prone position on the operating table and then started remifentanil infusion at a rate of $8 \mu \mathrm{g} / \mathrm{kg} / \mathrm{min}$ throughout the surgery. Anesthetics were modified to maintain hemodynamic variables within $20 \%$ of baseline values. At the start of the skin suturing, the anesthetic agents were discontinued and residual neuromuscular blockade was antagonized by administering $4 \mathrm{mg} / \mathrm{kg}$ sugammadex sodium. Patients were extubated when they met the tracheal extubation criteria (respiratory rate $>10$, spontaneous breathing with a volume of $10 \mathrm{ml} /$ $\mathrm{kg}$ sustained arm lift). At the end of the operation all patients were transferred from the OR to PACU.

All patients were discharged from PACU according to the modified Aldrete recovery score and the ability to move the blocked extremity. Patients who scored $>9$ were transferred to the regular ward (Table I).

\section{Parameters}

For all patients, we recorded and evaluated the demographic parameters, BMI, ASA scores, perioperative hemodynamics, time elapsed from OR entry until incision, operative time, time elapsed from application of the surgical dressing to exiting OR, intraoperative blood loss, PACU time, preoperative and postoperative $12^{\text {th }}$ hour pain score, postoperative analgesic requirements, first mobilization time, first oral intake, length of hospital stay and time to return to work. The time to return to work or daily activities for all patients was obtained via phone calls and recorded. Additionally, we calculated the perioperative anesthetic costs. Before discharging the patients from the hospital; the surgeons, anesthesiologists and patients answered questionnaires to determine their satisfaction (poor, good, or very good).

We recorded the heart rate, mean arterial blood pressure, and peripheral oxygen saturation before anesthesia; every 5 minutes during the operation; at the end of the surgery, and 60 minutes, 120 minutes, and 24 hours after the operation. We recorded the amount of blood loss from the surgical

Table I: Modified Aldrete Score (18)

\begin{tabular}{|c|c|}
\hline Criteria & Score \\
\hline \multicolumn{2}{|l|}{ 1. Activity } \\
\hline Moves all extremities & 2 \\
\hline Moves two extremities & 1 \\
\hline Unable to move extremities & 0 \\
\hline \multicolumn{2}{|l|}{ 2. Respiration } \\
\hline Breathes deeply, coughs freely & 2 \\
\hline Dyspneic, shallow or limited breathing & 1 \\
\hline Apneic & 0 \\
\hline \multicolumn{2}{|l|}{ 3. Circulation (blood pressure) } \\
\hline $20 \% \pm$ preanaesthetic level & 2 \\
\hline $20-49 \% \pm$ preanaesthetic level & 1 \\
\hline $50 \% \pm$ preanaesthetic level & 0 \\
\hline \multicolumn{2}{|l|}{ 4. Consciousness } \\
\hline Fully awake & 2 \\
\hline Arousable on calling & 1 \\
\hline Not responding & 0 \\
\hline \multicolumn{2}{|l|}{ 5. Oxygen saturation } \\
\hline $\mathrm{SpO}_{2}>92 \%$ on room air & 2 \\
\hline $\begin{array}{l}\text { Supplemental oxygen requirement to } \\
\text { maintain } \mathrm{SpO}_{2}>90 \%\end{array}$ & 1 \\
\hline $\begin{array}{l}\mathrm{SpO}_{2}<90 \% \text { with oxygen } \\
\text { supplementation }\end{array}$ & 0 \\
\hline
\end{tabular}


site by calculating the suctioned blood volume and the weight of bloody gauze. The patients were observed for postoperative complications in PACU and the intraoperative and postoperative complications were recorded.

Numeric Pain Rating Scale (NPRS) was used to measure the pain intensity. The patients were asked to rate their pain from 0 to 10 on a 10-point linear scale, zero indicating = no pain and 10 indicating = the worst pain. We recorded NPRS scores before and 12 hours after the operation. When the NPRS score was found to be greater than 4 , we administered meperidine $0.5 \mathrm{mg} / \mathrm{kg}$ as a rescue medication. If the scores did not improve after 30 minutes, we administered an additional dose of meperidine $0.2 \mathrm{mg} / \mathrm{kg}$.

We defined the operative time as the time taken from incision to application of the surgical dressing.

\section{Statistical Analysis}

Data were analyzed using Number Cruncher Statistical System (NCSS) 2007 (Kaysville, UT, USA), and the variables were presented as means \pm standard deviation or number. Between group comparisons were performed for age, weight, height, mean blood pressure, heart rate, operative time, length of hospital stay, and blood loss using Student's t-test. Continuous data were analyzed using Mann-Whitney Utest, Fisher-Freeman-Halton exact test, and Student's t- tests. Gender, ASA physical status, patient and surgeon satisfaction, postoperative analgesic use, and comorbidities were compared using Fisher's exact and Pearson's chisquared tests. We considered $p$ values $<0.05$ as significant.

\section{口 RESULTS}

No significant demographic differences were found between the two groups regarding the age or gender ( $p>0.05)$. BMI was noted to be $29.15 \pm 5.37 \mathrm{~kg} / \mathrm{m}^{2}$ in the GA group; and; 27.33 $\pm 3.69 \mathrm{~kg} / \mathrm{m}^{2}$ in the SA group, $(p=0.052)$ and no intergroup difference was found.

According to the ASA classifications, 38\% (n=38) of the patients were as having ASA physical status I, $58 \%(n=58)$ as having ASA physical status II and $4 \%(n=4)$ as having physical status ASA III (Table II).

We found the heart rate and the mean arterial pressure to be lower in the SA group than in the GA group (Table III).

We found no intergroup differences regarding the preoperative NPRS scores $(p=0.400)$. However, the postoperative NPRS scores were significantly lower in the SA group than in the GA group $(p=0.001)$ (Table IV).

The OR time until incision was $23.66 \pm 3.25$ minutes for the GA group; and $8.46 \pm 1.09$ minutes for the SA group, $(p=0.001)$.

The operative time was $2.13 \pm 0.84$ hours in the GA group and $1.72 \pm 0.49$ hours in SA group, $(p=0.008)$.

Time elapsed from surgical dressing to exiting OR was 19.94 \pm 2.5 minutes for the GA group and $10.34 \pm 1.89$ minutes for the SA group, $(p=0.001)$.
The mean estimated blood loss was lesser in the SA group than in the GA group $(p=0.001)$. The postoperative analgesic use was higher in the GA group than in the SA group $(p=0.001)$.

We observed earlier mobilization in the SA group than in the GA group $(p=0.001)$. The first oral intake occurred earlier in the patients in the SA than in those in the GA group $(p=0.001)$. For a comparative evaluation of patients' length of hospital stay, we performed hourly instead of daily calculation. Length of hospital stay was found to be $36.48 \pm 15.52$ hours for the GA group, $24.48 \pm 3.39$ hours for the SA group $(p=0.001)$.

The time to return to work or daily activities was $26.22 \pm$ 16.62 days in the GA group, and $14.50 \pm 16.28$ days in the SA group $(p=0.001)$. The cost of anesthesia was lower in the SA group at $10.40 \pm 1.11$ Turkish Liras $(T L)(p=0.001)$ than in the GA group $210.00 \pm 88.43 \mathrm{TL}(p=0.001)$. Finally, the patients', anesthesiologists', and surgeons satisfaction were significantly higher in the SA group than in the GA group $(p=0.001)$ (Table V). We encountered no complications in any of the patients in either study groups.

\section{- DISCUSSION}

GA is a widely accepted anesthetic technique for lumbar spine surgeries, despite no reasonable evidence yet supporting its superiority over SA. This preference is partly because of the surgeons' and anesthesiologists' lack of experience and knowledge regarding the benefits of regional anesthesia (6).

Notably, our results revealed better perioperative outcomes in the patients in the SA group than in those in the GA group. The advantages of SA included; better hemodynamic changes, shorter hospitalization time and quicker return to work, lesser time elapsed from OR entry until incision and operative time, lesser blood loss and postoperative analgesic requirements, better postoperative NPRS scores, earlier postoperative mobilization and first oral intake, cheaper anesthesia-related costs, and higher satisfaction among the patients surgeons and anesthesiologists.

The parameters indicating hemodynamic changes are affected by anesthesia, specifically GA. Although Vural and Yorukoglu reported no differences in the hemodynamic parameters after GA and SA (20), most studies have reported better hemodynamic outcomes with SA as consistent with our results $(2,3,5,7,9,14,17)$.

Our study revealed that SA shortens both time to surgery in OR (time elapsed from OR entry until incision), and operating time (skin to skin surgery time). These findings are concordant with those of few previous reports $(8,10,21)$.

The time elapsed from OR entry until incision is critical for most of the neurosurgeons who desire to start the procedure as soon as possible.

Moreover, SA has been reported to be associated with less intraoperative blood loss than GA $(3,5,11)$. A randomized prospective study conducted by Attari et al. showed that SA was superior to GA in terms of decreasing intraoperative blood loss and yielding better hemodynamics (2). The reduction in 
Table II: Demographic Parameters, BMI, and ASA Scores

\begin{tabular}{|c|c|c|c|c|c|}
\hline & & \multicolumn{4}{|c|}{ Type of Anesthesia } \\
\hline & & GA $(n=50)$ & SA $(n=50)$ & Total $(n=100)$ & $\mathbf{p}$ \\
\hline \multirow{2}{*}{ Gender } & Female & $26(52 \%)$ & $24(48 \%)$ & $50(50 \%)$ & $0.689^{a}$ \\
\hline & Male & $24(48 \%)$ & $26(52 \%)$ & $50(50 \%)$ & \\
\hline Mean age & Mean \pm SD & $53.2 \pm 3.1$ & $54.1 \pm 2.4$ & & $0.051^{b}$ \\
\hline \multirow{2}{*}{ BMI $\left(\mathrm{kg} / \mathrm{m}^{2}\right)$} & Min-Max (range) & $19.8-51,9$ & $20.6-34.4$ & & $0.052^{b}$ \\
\hline & Mean \pm SD & $29.15 \pm 5.37$ & $27.33 \pm 3.69$ & $28.24 \pm 4.67$ & \\
\hline \multirow{3}{*}{ ASA } & ASA I & $20(20 \%)$ & $18(18 \%)$ & $38(38 \%)$ & $0.004^{\star * c}$ \\
\hline & ASA II & $28(28 \%)$ & $30(30 \%)$ & $58(58 \%)$ & \\
\hline & ASA III & $2(2 \%)$ & $2(2 \%)$ & $4(4 \%)$ & \\
\hline
\end{tabular}

${ }^{a}$ Pearson's chi-squared $t$ tests, ${ }^{b}$ Student $t-T e s t,{ }^{c}$ Fisher-Freeman-Halton exact Test, ${ }^{*} p<0.05,{ }^{* *} p<0.01$, BMI: Body mass index, ASA: American Society of Anesthesiologists.

Table III: Perioperative and Physiological Characteristics

\begin{tabular}{|c|c|c|c|}
\hline \multirow{2}{*}{ Characteristic } & \multicolumn{2}{|c|}{ Type of Anesthesia } & \multirow[b]{2}{*}{$\mathbf{p}$} \\
\hline & GA & SA & \\
\hline Preoperative MAP (SD) & 102.8 & 90.2 & $0.74^{a}$ \\
\hline Preoperative HR (SD) & 72.9 & 72.5 & $0.23^{a}$ \\
\hline Intraoperative MAP (SD) & 80.6 & 60.7 & $0.09^{a}$ \\
\hline Intraoperative mean HR (SD) & 79.0 & 56.6 & $0.014^{\mathrm{a}}$ \\
\hline Postoperative MAP (SD) after $1 \mathrm{~h}$ & 85.6 & 68.9 & $0.005^{\mathrm{a}}$ \\
\hline Postoperative mean HR (SD) after $1 \mathrm{~h}$ & 80.2 & 67.8 & $0.001^{\mathrm{a}}$ \\
\hline Postoperative MAP (SD) after one day & 80.6 & 64.9 & $0.005^{\mathrm{a}}$ \\
\hline Postoperative mean HR (SD) after one day & 80.0 & 60.0 & $0.001^{a}$ \\
\hline
\end{tabular}

a Student's t-Test, MAP: Mean arterial pressure, HR: Heart rate.

Table IV: Preoperative and Postoperative NPRS Scores

\begin{tabular}{|c|c|c|c|c|c|}
\hline & & \multirow[b]{2}{*}{$\mathrm{GA}(\mathrm{n}=50)$} & \multicolumn{2}{|c|}{ Type of Anesthesia } & \multirow{2}{*}{$\mathbf{p}^{a}$} \\
\hline & & & SA $(n=50)$ & Total $(n=100)$ & \\
\hline \multirow{2}{*}{ Preoperative NPRS } & Min/Max (median) & 4 / $10(8)$ & $4 / 10(7.5)$ & 4 / $10(8)$ & 0.400 \\
\hline & Mean \pm SD & $7.44 \pm 1.30$ & $7.16 \pm 1.54$ & $7.30 \pm 1.42$ & \\
\hline \multirow{3}{*}{$\begin{array}{l}\text { Postoperative NPRS } \\
\text { after } 12 \text { hours }\end{array}$} & Min/Max (median) & 0 / $4(2)$ & $0 / 4(1)$ & $0 / 4(1)$ & $0.001^{* *}$ \\
\hline & Mean \pm SD & $1.68 \pm 0.89$ & $0.92 \pm 0.99$ & $1.30 \pm 1.01$ & \\
\hline & $\mathbf{p}^{\mathbf{b}}$ & $0.001^{* *}$ & $0.001^{* *}$ & & \\
\hline \multirow{2}{*}{ Preop-Postop difference } & Min/Max (median) & $-8 /-2(-6)$ & $-9 /-2(-6)$ & $-9 /-2(-6)$ & 0.044 \\
\hline & Mean \pm SD & $-5.76 \pm 1.13$ & $-6.24 \pm 1.49$ & $-6.00 \pm 1.34$ & \\
\hline
\end{tabular}

a Mann Whitney $U$ Test, ${ }^{b}$ Wilcoxon Signed Ranks Test, ${ }^{* *} p<0.01$, NPRS: Numeric pain rating scale. 
Table V: Intraoperative and Postoperative Outcomes in General and Spinal Anesthesia Groups

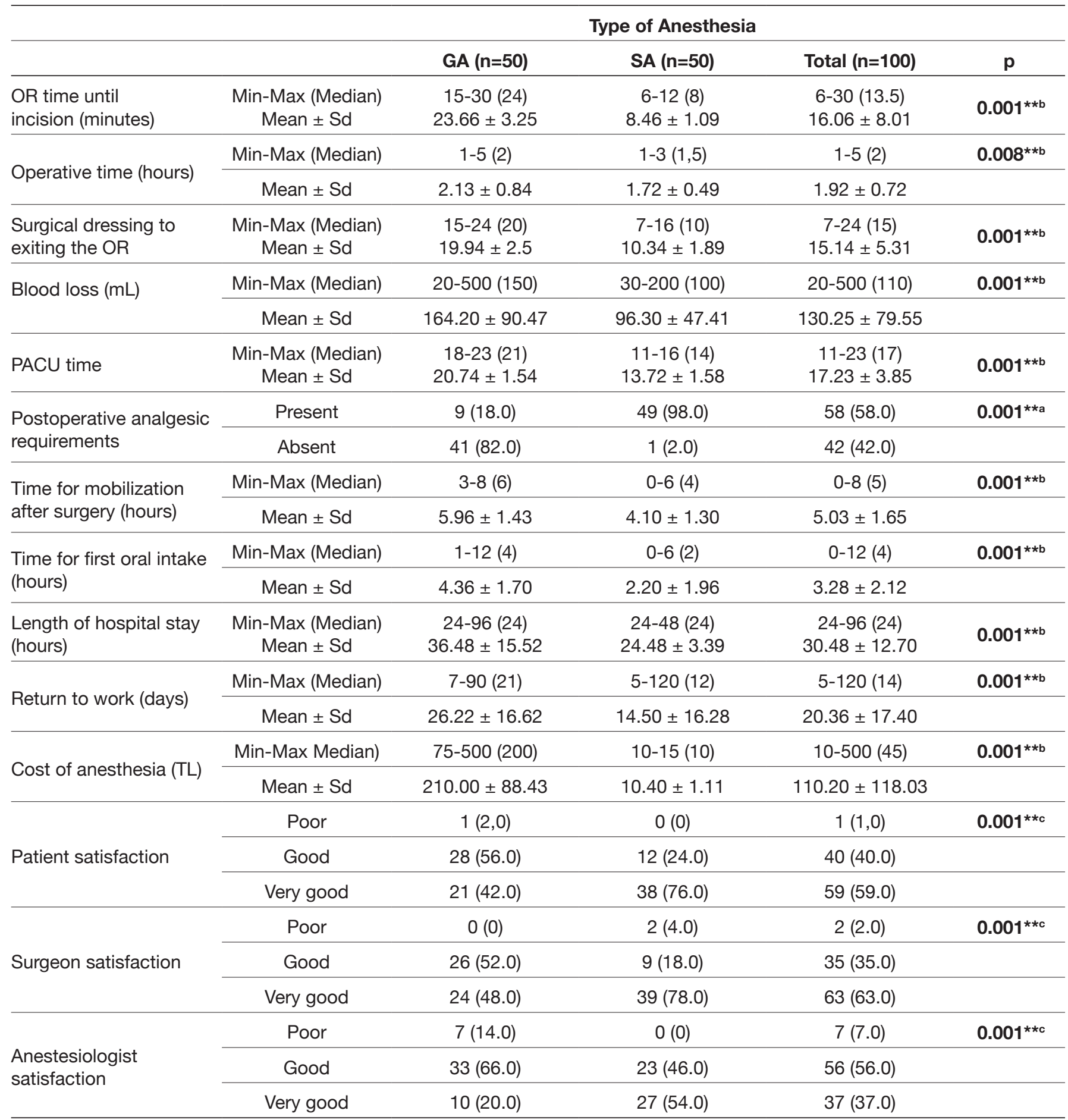

${ }^{a}$ Pearson chi-squared Test, ${ }^{b}$ Mann Whitney $U$ Test, ${ }^{c}$ Fisher-Freeman-Halton exact Test, ${ }^{* *} p<0.01$, TL: Turkish Liras, OR: Operating room, PACU: Post anesthetia care room. 
blood loss may be attributable to vasodilation after SA. SA also causes a sympathetic block and lowers the intrathoracic pressure. All these phenomena result in lesser distension of the epidural veins.

The reduced blood loss and controlled hypotension under SA may be the reasons behind the shorter operation times. Notably, our study confirmed shorter operation times in the SA group $(7,10,21)$.

Postoperative pain and pain control are essential in patients undergoing microdiscectomy. The residual sensory block after SA helps in the recovery of patients. The inhibition of the afferent nociceptive pathways may decrease the reported pain scores and after SA compared with those after GA $(2,5,7,9,10,12,17)$. Notably, lower pain scores require lesser postoperative analgesia $(2,4,7,8-10,17,20)$, as confirmed by our results. We firmly believe that the lower pain scores noted in the SA group in our study may have resulted in a quicker postoperative mobilization and oral intake compared with the GA group.

The length of hospital stay has been reported to be shorter in the SA group $(10,11)$, or similar to that of the GA group $(4,5,20)$. The shorter hospitalization times of patients under SA may be because of the lower complication rates. Moreover the patients to be discharged first in our study belonged to the SA group and consequently they had a quicker return to work.

The two other advantages of SA over GA found in our study were the reduced anesthesia cost and the higher surgeon and patient satisfaction.

The cost of anesthesia for the SA group has been reported to be lower than for the GA group $(1,7,17,20,21)$, and our study results were concordant with this finding. Walcott et al. performed a cost comparison analysis of SA versus GA for lumbar spine surgeries by calculating the OR costs and found significantly reduced costs of SA compared with those of GA $(1,21)$.

We also found out that the cost for SA is lower than that for GA because the cost of the SA medications is lower than that of the GA medications. The volatile gas, total intravenous medications and the instruments used during GA are expensive. Therefore SA is an economical alternative in well selected patients.

Another critical variable is satisfaction among the surgeons and patients determined using a simple scale; we found increased satisfaction after SA based on a simple assessment.

Vural and Yorukoglu has reported better patient satisfaction and Attari et al. have reported better surgeon satisfaction with SA $(2,20)$.

\section{CONCLUSION}

SA is a safe and inexpensive anesthetic option with superior results. A shorter time elapsed from OR entry until incision, a shorter hospital stay, and a shorter time to return to work are the major benefits of SA over GA. Additionally, SA is considered satisfactory by the patients, anesthesiologists, and surgeons.
Therefore, based on our results, SA can be considered for use as the primary anesthetic option for patients undergoing lumbar microdiscectomy.

\section{- REFERENCES}

1. Agarwal P, Pierce J, Welch W: Cost analysis of spinal versus general anesthesia for lumbar diskectomy and laminectomy spine surgery. World Neurosurg 89:266-271, 2016

2. Attari MA, Mirhosseini SA, Honarmand A, Safavi MR: Spinal anesthesia versus general anesthesia for elective lumbar spine surgery: A randomized clinical trial. J Res Med Sci 16:524-529, 2011

3. Dagistan Y, Okmen K, Dagistan E, Guler A, Ozkan N: Lumbar microdiscectomy under spinal and general anesthesia: $A$ comparative study. Turkish Neurosurgery 25:685-689, 2015

4. De Rojas JO, Syre P, Welch WC: Regional anesthesia versus general anesthesia for surgery on the lumbar spine: A review of the modern literature. Clin Neurol Neurosurg 119:39-43, 2014

5. Demirel CB, Kalayci M, Ozkocak I, Altunkaya H, Ozer $\mathrm{Y}$, Acikgoz B: A prospective randomized study comparing perioperative outcome variables after epidural or general anesthesia for lumbar disc surgery. J Neurosurg Anesthesiol 15:185-192, 2003

6. Hassi N, Badaoui R, Cagny-Bellet A, Sifeddine S, Ossart M: Spinal anesthesia for disc herniation and lumbar laminectomy. Apropos of 77 cases. Can Anesthesiol 43:221-225, 1995

7. Kahveci K, Doger C, Ornek D, Gokcinar D, Aydemir S, Ozay R: Perioperative outcome and cost-effectiveness of spinal versus general anesthesia for lumbar spine surgery. Neurologia i Neurochirurgia Polska 48:167-173, 2014

8. Kara I, Celik JB, Bahar OC: Comparison of spinal and general anesthesia in lumbar disc surgery. Journal of Neurological Sciences (Turkish) 28:487-496, 2011

9. Karaman S, Karaman T, Dogru S: Retrospective evaluation of anesthesia approaches for lumbar disc surgery. Journal of Anesthesia and Clinical Research 5:4, 2014

10. McLain RF, Tetzlaff JE, Bell GR, Uwe-Lewandrowski K, Yoon HJ, Rana M: Microdiscectomy: Spinal anesthesia offers optimal results in general patient population. J Surg Orthop Adv 16:5-11, 2007

11. Meng T, Zhong Z, Meng L: Impact of spinal anesthesia vs. general anesthesia on perioperative outcome in lumbar spine surgery: A systematic review and meta analysis of randomised, controlled trials. Anaesthesia 72:391-401, 2017

12. Sadrolsadat SH, Mahdavi AR, Moharari RS, Khajavi MR, Khashayar P, Najafi A, Amirjamshidi A: A prospective randomized trial comparing the technique of spinal and general anesthesia for lumbar disk surgery: A study of 100 cases. Surg Neurol 71:60-65, 2009

13. Saraswat V: Effects of anaesthesia techniques and drugs on pulmonary function. Indian J Anaesth 59(9):557-564, 2015

14. Scott NB, Kehlet $\mathrm{H}$ : Regional anaesthesia and surgical morbidity. BJ Surg 75(4):299-304, 1988

15. Smrčka M, Baudysovå O, Jurån V, Vidlák M, Gál R, Smrčka $\mathrm{V}$ : Lumbar disc surgery in regional anesthesia: 40 years of experience. Acta Neurochir (Wien) 143:377-381, 2001 
16. Tarıkçı Kılıç E, Aydin G: Effects of dexmedetomidine infusion during spinal anesthesia on hemodynamics and sedation. Libyan J Med 13(1):1436845, 2018

17. Tetzlaff JE, Dilger JA, Kodsy M, al-Bataineh J, Yoon HJ, Bell GR: Spinal anesthesia for elective lumbar spine surgery. J Clin Anesth 10:666-669, 1998

18. Truong L, Moran JL, Blum P: Postanaesthesia care unit discharge: A clinical scoring system versus traditional time based criteria. Anaest Intensive Care 32(1):33-42, 2004

19. Tzovaras G, Fafoulakis F, Pratras K, Georgopoulou S, Stamatiou G, Hatzitheofilou C: Spinal vs general anesthesia for laparoscopic cholecystectomy: Interim analysis of a controlled randomized trial. Arch Surg 143:497-501, 2008
20. Vural C, Yorukoglu D: Comparison of patients satisfaction and cost in spinal and general anesthesia for lumbar disc surgery. Turkish Neurosurgery 24:380-384, 2014

21. Walcott BP, Khanna A, Yanamadala V, Coumans JV, Peterfreund RA: Cost analysis of spinal and general anesthesia for the surgical treatment of lumbar spondylosis. Journal of Clinical Neuroscience 22:539-543, 2015

22. Weinstein JN, Lurie JD, Tosteson TD, Skinner JS, Hanscom B, Tosteson AN, Herkowitz H, Fischgrund J, Cammisa FP, Albert T, Deyo RA: Surgical vs nonoperative treatment for lumbar disk herniation: The Spine Patient Outcomes Research Trial (SPORT) Observational Cohort. JAMA 296:2451-2459, 2006 